\title{
El enalapril también retarda la progresión de nefropatía en diabéticos normotensos y normoalbuminúricos
}

Use of enalapril to attenuate decline in renal function in normptensive, normoalbuminuric patients with type 2 diabetes mellitus. A randomized, controlled trial. Ravid M, Levi Z, Bar-Dayan Y et al. Ann Intern Med 1998;128:982-88

\section{Objetivo}

Evaluar el efecto del tratamiento prolongado con enalapril en la función renal y la albuminuria en pacientes con diabetes tipo 2 (DBT 2).

\section{Diseño}

Estudio randomizado doble ciego, controlado con placebo. Seguimiento a seis años.

\section{Lugar}

Ocho centros ambulatorios coordinados por el departamento de medicina de un hospital universitario, Israel.

\section{Pacientes}

Se incluyeron 156 pacientes con diagnóstico de DBT 2 luego de los 40 años con tensión arterial y albuminuria normales. La tensión arterial media promedio era $<107 \mathrm{mmHg}$ y la excreción de albúmina $<30 \mathrm{mg} / 24 \mathrm{hs}$.

\section{Intervención}

Enalapril, $10 \mathrm{mg} /$ día vs. placebo.

\section{Medición de resultados principales}

Albuminuria de 24 hs, clearence de creatinina, tensión arterial, y hemoglobina glicosilada (HbA1c).

\section{Resultados principales}

\begin{tabular}{l|lllll}
\hline Variable & $\begin{array}{l}\text { Grupo } \\
\text { Enalapril }\end{array}$ & $\begin{array}{l}\text { Grupo } \\
\text { Placebo }\end{array}$ & $p$ & $\begin{array}{c}\text { RRA } \\
\text { (IC 95\%) }\end{array}$ & $\begin{array}{c}\text { NNT } \\
\text { (IC 95\%) }\end{array}$ \\
\hline $\begin{array}{l}\text { Albuminuria inicial } \\
\text { (SD) } \mathrm{mg} / \mathrm{d}\end{array}$ & $11.6(7)$ & $10.8(8)$ & NS & & \\
$\begin{array}{l}\text { Albuminuria a los } \\
6 \text { años (SD) }\end{array}$ & $15.8(8)$ & $26.5(10)$ & 0.001 & & \\
\hline $\begin{array}{l}\text { Desarrollo de } \\
\text { microalbuminuria } \\
\text { a los } 6 \text { años }\end{array}$ & $6.5 \%$ & $19 \%$ & 0.04 & $\begin{array}{c}12.5 \% \\
(2-23 \%)\end{array}$ & $\begin{array}{c}8 \\
(4-50)\end{array}$ \\
\hline
\end{tabular}

El tratamiento con enalapril redujo la albuminuria de 24 hs. de $11.6+/-$ $7 \mathrm{mg} / \mathrm{d}$ al inicio a $9.7+/-6 \mathrm{mg} / \mathrm{d}$ a los dos años. Luego la albuminuria aumentó gradualmente hasta llegar a $15.8+/-8 \mathrm{mg} / \mathrm{d}$ a los seis años. En el grupo placebo la albuminuria aumentó de $10.8+/-8$ a $26.5+/-10 \mathrm{mg} / \mathrm{d}$ a los seis años. La HbA1c se redujo modestamente en ambos grupos, y en ambos grupos se mantuvo la normotensión. La declinación anual promedio del clearence de creatinina fue de $0.04 \mathrm{ml} / \mathrm{s}$ en el grupo placebo y de 0.025 en el grupo enalapril ( $p$ significativa luego del cuarto año).

\section{Conclusiones}

El enalapril atenúa la declinación de la función renal y reduce el grado de albuminuria en pacientes con DBT 2 normotensos y normoalbuminúricos.

\section{COMENTARIO}

La microalbuminuria es un precoz marcador de nefropatía en los pacientes diabéticos. Es un predictor independiente de progresión de nefropatía, así como de morbimortalidad cardiovascular ${ }^{1}$.

Los inhibidores de la enzima convertidora de angiotensina (IECA) han demostrado retardar la progresión de nefropatía en pacientes diabéticos con microalbuminuria inicial, ya sean normo o hiperten$\operatorname{sos}^{2-5}$. La limitación más seria de este trabajo es la imposibilidad de realizar un análisis por intención de tratar* ya que no tuvieron un seguimiento completo de los pacientes que abandonaron el estudio (de todos modos, estos fueron 38 pacientes de los 194 randomizados originalmente, con características similares a los que finalizaron el estudio y distribuidos en ambos grupos de manera similar). El aporte de este estudio es contestar la siguiente pregunta: ¿Son eficaces los IECA en pacientes con DBT2 normotensos y normoalbuminúricos? La respuesta de este estudio es afirmativa: el enalapril retarda la progresión de microalbuminuria comparado con el placebo. La siguiente pregunta es: ¿cuál es la magnitud del efecto? Para evaluar esto hay \# final un resultado intermedio* (microalbuminuria). A pesar de que la inicroalbuminuria puede evolucionar a largo plazo (más de diez años en promedio) a insuficiencia renal, este resultado final* no es evaluado en el trabajo (para ello serían necesarios más pacientes o más años de seguimiento). Teniendo esto en cuenta, hacen falta tratar a 8 pacientes con DBT2 normotensos, normoalbuminúricos por un periodo de seis años para prevenir que uno desarrolle microalbuminuria, pero no podemos inferir directamente su efecto en el desarrollo de insuficiencia renal. Este efecto es modesto si se lo compara al tratamiento con IECA en pacientes con microalbuminuria o nefropatía declarada ${ }^{5-7}$

Es importante destacar también que el grupo enalapril tuvo una tensión arterial menor que el placebo, y no se puede saber con certeza cuál es el mecanismo de protección (si es el enalapril por sí mismo o si es por el hecho de bajar la tensión arterial).

En conclusión, este estudio muestra un beneficio modesto y estadísticamente significativo de los IECA en la protección de nefropatía en DBT2 normotensos y normoalbuminúricos y con un similar control glucémico (HbA1C similares en ambos grupos). Son necesarios estudios a más largo plazo para observar si este efecto persiste y retarda la nefropatía manifiesta. Hasta ese momento, la indicación de IECA en estos diabéticos de bajo riesgo debe ser individualizada.

*Ver Glosario

\section{Dr. Federico Augustovski}

Unidad de Medicina Familiar y Preventiva. Hospital Italiano de Buenos Aires.

Inimeen SF, Gerstein H. The association of microalbuminuria and mortality in non-insulin dependent diabetes mellitus. A systematic overview of the literature. Arch Intern Med $=157: 1413-18$

Fijurar, Mulec H, Johnsen SA y cols. Renal protective effect of enalapril in diabetic nephropathy. BMJ 1992;304:339-43

(awis E, Hunsicker LG, Bain RP, y cols. The effect of angiotensin converting enzyme inhitition on diatic nephropathy.

wartivesen ER, Hommel E, Giese J y cols. Efficacy of angiotensin converting enzyme inhibition on diabetic nephropathy. The Collaborative Study Group. N Engl J Med 1993;329:1456-62

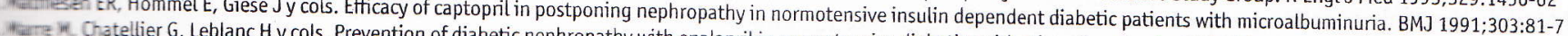

warre $)$ Chatellier $\mathrm{G}$, Leblanc $\mathrm{H}$ y cols. Prevention of diabetic nephropathy with enalapril in normotensive diabetics with microalbuminuria. BMJ 1988:297:1092-5

Wesurz HE, Wiegmann TB, Cnaan A, y cols. Renal protective effect of enalapril in hypertensive NIDDM: role of baseline albuminuria. Kidney Int Suppl 1994:45:S150-5

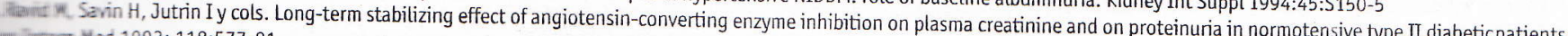
Ninn Iintern Med 1993; 118:577-81 